\title{
Analysis of the association of chronic spontaneous urticaria with interlekin-4, -10, transforming growth factor- $\beta 1$, interferon- $\gamma$, interleukin-17A and -23 by autologous serum skin test
}

\author{
${ }^{1}$ Allergy Immunology Department, Tepecik Training and Research Hospital, Izmir, Turkey \\ ${ }^{2}$ Division of Allergy Immunology, Department of Internal Medicine, Celal Bayar University, Manisa, Turkey \\ ${ }^{3}$ Department of Histology and Embryology, Faculty of Medicine, Celal Bayar University, Manisa, Turkey \\ ${ }^{4}$ Department of Biochemistry, Faculty of Medicine, Celal Bayar University, Manisa, Turkey \\ ${ }^{5}$ Department of Public Health, Faculty of Medicine, Celal Bayar University, Manisa, Turkey
}

Papatya Bayrak Degirmenci ${ }^{1}$, Cengiz Kırmaz ${ }^{2}$, Seda Vatansever ${ }^{3}$, Ece Onur ${ }^{4}$, Emine $\mathrm{Nal}^{3}$, Soner Erdin ${ }^{4}$, Beyhan Ozyurt ${ }^{5}$

Adv Dermatol Allergol 2016; XXXIV (1): 70-76

DOI: https://doi.org/10.5114/pdia.2016.57679

\begin{abstract}
Aim: To contribute to the understanding of the pathogenesis of chronic spontaneous urticaria (CSU) by identifying its relationship with autoimmunity and cytokines using the autologous serum skin test (ASST) and peripheral blood mononuclear cell culture (PBMC) method.

Material and methods: Interleukins (IL)-4, IL-10, transforming growth factor (TGF- $\beta 1$ ), interferon (IFN)- $\gamma$, IL-17A, and IL-23 levels in cell supernatants obtained by the PBMC method were measured using ELISA. Disease activity was assessed by determining the urticaria activity score (UAS).

Results: A total of 40 patients diagnosed with CSU participated in this study. Twenty patients had positive ASST results, and 20 had negative results. The control group included 20 healthy volunteers. We found that the IL-23 ( $p=0.01)$, IL-10 $(p=0.04)$ and IL-4 $(p=0.04)$ levels of the patient groups were significantly lower compared with those of the control group. The IL-23 ( $p=0.009)$, IL-10 ( $p=0.009)$, IL-4 ( $p=0.001)$, and IL-17 $(p=0.05)$ levels of the ASST(-) patient group were significantly lower compared with those of the control group. In addition, the IL-4 $(p=0.03)$ and IFN- $\gamma(p=0.05)$ levels of the ASST $(+)$ patient group were significantly lower compared with those of the control group, and the ASST(+) patients had a significantly higher UAS than the ASST $(-)$ patients $(p=0.021)$. Conclusions: These results, when considered together with current reports in the literature, indicate that immune dysregulation occurs in the pathogenesis of CSU, causing cytokine imbalance.
\end{abstract}

Key words: chronic spontaneous urticaria, cytokines, autologous serum skin test.

\section{Introduction}

Chronic spontaneous urticaria (CSU), also known as chronic idiopathic urticaria (CIU), is characterized by the spontaneous occurrence of wheals without an obvious stimulus that last for most days of the week for more than 6 weeks [1, 2].

The pathogenesis of CSU is unclear, and possible causes include acute or chronic infection, pseudo-allergies to food and drugs, allergic reactions to medications, food, or insect stings and bites and autoreactivity, including autoimmunity mediated by functional autoantibodies directed against immunoglobulin E (IgE) receptor [3].
An autoimmune etiology characterized by the presence of histamine-releasing autoantibodies directed against the subunit of high-affinity IgE receptor (FcERI) or IgE has been found in approximately $35-45 \%$ of CSU patients [4]. These autoantibodies can be detected in vivo by the autologous serum skin test (ASST) [5]. The sensitivity and specificity of the ASST for autoantibody detection in patients with CSU is reported to be $80 \%$ at best compared with the in vitro basophil histamine release assay [6]. The ASST is an in vivo screening test for detecting histaminereleasing autoantibodies and other vasoactive factors, and it has a high negative predictive value $[7,8]$.

Address for correspondence: Dr. Papatya Bayrak Degirmenci MD, Fatih Sultan Mehmet Caddesi, Seyhan Sitesi No: 29/1, Cblok D: 10, Bornova, Izmir, Turkey, phone: +90 05416549994, e-mail: papatyabayrak@yahoo.com Received: 3.08.2015, accepted: 26.01.2016. 
Histamine-releasing autoantibodies directed against FcERI or IgE are detected in only one-third of CSU cases, suggesting that other circulating mediators, including cytokines, may be involved in its pathophysiology [9-11]. Most autoimmune and/or allergic diseases are caused by an imbalance between cytokines and T lymphocyte subgroups. The CSU shows both autoimmune and allergic disease characteristics [12]. The determination of the dominant cytokine pattern and T lymphocyte subgroup in CSU patients may be key to explaining this complicated immunopathogenesis.

Various cytokines have been found to be involved in inflammatory processes associated with CSU. Thus, in our study we examined the levels of interleukin (IL)-4, IL-10, IL-17, IL-23, transforming growth factor (TGF)- $\beta$, interferon (IFN)- $\gamma$ which are associated with T lymphocyte subgroups.

Interleukin-4 plays a central role in the differentiation of antigen-stimulated naïve T cells into T helper (Th) 2 cells, which are the main sources of IL-4 [13]. Interleukin-4 also suppresses Th1 cell development and directs the switching of human B cells to IgE and IgG4 [14].

Transforming growth factor $\beta 1$ is a cytokine with diverse effects on cell function, differentiation, and proliferation. It also acts as an anti-inflammatory cytokine in the skin, mainly via the inhibition of mast cell survival and function, suppresses the expression of both FceRI and Ig-E on the mast cell surface, and attenuates mediator release from mast cells [15]. Transforming growth factor $\beta$ is the amplifying cytokine in regulatory $T$ (Treg) cells [16].

Interleukin-10, another anti-inflammatory cytokine, exerts similar effects, such as the inhibition of FcERI expression on mast cells and the suppression of inflammatory mediator release. Interleukin-10 is mainly produced by Treg cells [17-19].

Interferon- $\gamma$ is a multipotent cytokine that plays important roles in both the innate and adaptive immune responses. Interleukin-4, as well as IL-10, TGF- $\beta$, and glucocorticoids, negatively regulate the production of IFN- $\gamma$. High levels of this cytokine are expressed by Th1 cells, promoting the cytotoxic activities of other cells and inducing the apoptosis of epithelial cells in the skin and mucosa [20-22].

Interleukin-17A is another proinflammatory cytokine that is mainly expressed by fibroblasts and Th17 cells. Its role in neutrophil recruitment is crucial for host protection against various extracellular pathogens, such as bacteria and fungi. It is also important in autoimmunity and inflammation $[23,24]$.

Interleukin-23 is dispensable for the in vitro polarization of naïve T cells into Th17 cells, which produce IL-17. Interleukin-23 plays important roles in the functioning and continuity of the Th17 subtype [25]. Interleukin-23 may play a critical role in driving the early inflammatory immune response to pathogens or injury by directly inducing IL-17 production and early neutrophil recruitment.

Dysregulation of the IL-23/IL-17 immune axis has been linked to immunopathology and autoimmune inflammation [26, 27].

\section{Aim}

The main objective of this study was to contribute to the understanding of the pathogenesis of CSU by identifying its relationship with autoimmunity, T lymphocyte subgroups and cytokines using the ASST and peripheral blood mononuclear cell culture (PBMC) method. A secondary objective was to obtain results that contribute to the development of new treatment approaches.

\section{Material and methods}

This prospective study was approved on 07/05/2012 with decision number 2012/013 by the Ethical Board for Clinical Research at Celal Bayar University, Faculty of Medicine.

Written informed consent was obtained from all patients. The participants of this study were patients with symptoms of chronic urticaria who had been treated at the Internal Diseases Allergy Immunology Clinic at Celal Bayar University. The patients' medical histories were taken, and physical examination was performed. Routine clinical examinations related to chronic urticaria were performed, including the following: liver function tests; determination of the complete blood count, erythrocyte sedimentation rate, serum free $\mathrm{T} 4, \mathrm{~T} 3$, and thyroid-stimulating hormone (TSH) levels and anti-thyroglobulin (antiTG) and antimicrosomal antibody (anti-TPO) levels; an autoimmunity panel (ANA, anti-DNA, and RF); an infection panel (HBsAg, antiHBs, antiHCV, a parasite stool test, and complete urine analysis); a skin prick test for food and inhalant allergens; and provocation tests for physical urticaria (an ice test for cold urticaria or skin scratching for dermographism). All patients had active urticaria at the time of the study and were being treated with $\mathrm{H} 1$ antihistamines. Long-acting antihistamines were stopped at 7 days, and short-acting antihistamines were stopped at 3 days before blood samples were collected for the ASST, PBMC and ELISA.

The urticaria activity score (UAS) was used for the assessment of disease activity in all patients. It was calculated as recommended by the EAACI/GA2LEN/EDF/ WAO Guidelines [28]. The weekly UAS was estimated according to the number of wheals and pruritus intensity, which appeared during the week before the day of blood sampling. The patients were asked to record 24-h selfevaluation scores for 7 days, using the following scoring scheme: no wheals $=0 ;<20$ wheals $/ 24 \mathrm{~h}=1 ; 20-50$ wheals $/ 24 \mathrm{~h}=2$; and $>50$ wheals $/ 24 \mathrm{~h}=3$; and pruritus intensity: no $=0$; mild $=1$; moderate $=2$; and severe $=3$. 
The weekly UAS was equal to the sum of the scores obtained on 7 consecutive days, with a minimum score of 0 and a maximum score of 42 . Consequently, this score was graded as follows: 0-14 (mild), 15-29 (moderate) or 30-42 (severe).

\section{Autologous serum skin test}

A total of 10 cc of venous blood was collected from the patients into sterile plain tubes and left to coagulate for $30 \mathrm{~min}$ at room temperature. The blood was then centrifuged for $15 \mathrm{~min}$ at $500 \mathrm{~g}$, and the serum was eluted. A total of $50 \mu \mathrm{l}$ of the serum was drawn into an insulin injector and injected into the volar face of the forearm intradermally. Ten microliters of histamine (Allergopharma, Germany) as a positive control and $50 \mu \mathrm{l}$ of physiological saline solution as a negative control (for the intracutaneous test; Allergopharma, Germany) were injected intradermally at $5 \mathrm{~cm}$ from the area that received the autologous serum injection. After 30 min, erythema and induration were evaluated. The widest diameter of induration and the diameter that was perpendicular to this diameter were measured. Erythema and induration were graded as recommended by the EAACI/GA2LEN/ EDF/WAO Guidelines [7] as follows: grade 0: skin rash caused by physiological saline solution that is expected to be light pink or skin-colored; grade 1: the formation of pink color caused by autologous serum, whereas the area where physiological saline solution was injected remains skin-colored; and grade 2: the formation of a histaminelike red color caused by autologous serum, whereas the area where physiological saline solution was injected remains skin-colored. A positive test reaction was indicated when the erythema caused by the autologous serum was grade 2 and the diameter of induration was $1.5 \mathrm{~mm}$ greater than that of the induration caused by the physiological saline solution. All ASSTs were performed by the same researcher.

\section{Peripheral blood mononuclear cell culture method}

A volume of 15 cc of venous blood was collected from each patient to culture PBMCs. Peripheral blood mononuclear cells culture were prepared by standard Ficoll-Paque density gradient centrifugation [29]. Blood samples for PBMC cultures were poured into $50 \mathrm{ml}$ tubes, and an equal volume of phosphate- buffered solution (PBS) was added. The tubes were centrifuged for $20 \mathrm{~min}$ at $2300 \mathrm{rpm}$. Grey-white-colored cells that appeared at the junction of the PBS and Biocoll (L-6115, Biochrom) at the end of centrifugation were transferred into a new $15 \mathrm{ml}$ tube, to which an equal volume of PBS was added. Then, the mixture was centrifuged for $10 \mathrm{~min}$ at $2000 \mathrm{rpm}$, the pellets were washed with $1 \mathrm{ml}$ of PBS, and the tube was centrifuged at $1300 \mathrm{rpm}$ for $5 \mathrm{~min}$. The pellets were cultured in RPMI-1640 (BE12-167F, Lonza) medium containing 1\% L-glutamine (BE17-605E, Lonza), 10\% fetal bovine serum (S-0113, Biochrom) and 1\% penicillin/streptomycin (DE17-602E, Lonza). Cells $(3 \times 106$ cells $/ \mathrm{ml})$ were added to 6 wells of 24-well culture plates. Three of the wells were used as controls, to which only culture medium was added. Phytohemagglutinin (1\%; M5030, Biochrom) was added to the culture medium in the remaining three wells, representing the study group. Cells in both the control and study groups were cultured for $48 \mathrm{~h}$ at $37^{\circ} \mathrm{C}$ and $5 \% \mathrm{CO}_{2}$ in air. Then, the culture media and cells were collected for both the control and study groups and centrifuged at $1000 \mathrm{rpm}$ for $5 \mathrm{~min}$. Supernatants from the control and study groups were stored at $-20^{\circ} \mathrm{C}$ until cytokine measurements. To determine cell viability, the pellets were resuspended in $1 \mathrm{ml}$ of culture medium. Forty microliters of sample was mixed with the same amount of trypan blue, and staining and non-staining cells were calculated. The percentages of stained cells were $1 \%$ and $0.5 \%$ for the control and study groups, respectively.

\section{Cytokine measurements}

The IL-4, IL-10, TGF- $\beta 1$, IFN- $\gamma$, IL-17 and IL-23 levels in the cell supernatant from the PBMC were measured by ELISA.

Cell culture IL-4 concentrations were assayed using an enzyme-linked immunosorbent assay (ELISA) kit from Biosource (Nivelles, Belgium) in duplicate, and the mean of the two measurements was used as the final result. The analytical sensitivity of IL-4 was $2 \mathrm{pg} / \mathrm{ml}$, and the intraassay coefficients of variation were $3.0 \%$ for $47.9 \mathrm{pg} / \mathrm{ml}$ and $2.9 \%$ for $119.3 \mathrm{pg} / \mathrm{ml}$. The interassay coefficients of variation were $3.9 \%$ for $48.8 \mathrm{pg} / \mathrm{ml}$ and $4.4 \%$ for $119.1 \mathrm{pg} / \mathrm{ml}$.

Cell culture IL-10 concentrations were assayed using an ELISA kit from Biosource (Nivelles, Belgium) in duplicate, and the mean of the two measurements was used as the final result. The analytical sensitivity of IL-10 was $1 \mathrm{pg} / \mathrm{ml}$, and the intraassay coefficients of variation were $2.9 \%$ for $57.6 \mathrm{pg} / \mathrm{ml}$ and $2.9 \%$ for $138 \mathrm{pg} / \mathrm{ml}$. The interassay coefficients of variation were $2.9 \%$ for $55.7 \mathrm{pg} / \mathrm{ml}$ and $2.8 \%$ for $140.3 \mathrm{pg} / \mathrm{ml}$.

Cell culture TGF- $\beta 1$ concentrations were assayed using an ELISA kit from Biosource (Nivelles, Belgium) in duplicate, and the mean of the two measurements was used as the final result. The analytical sensitivity of TGF- $\beta 1$ was $15.6 \mathrm{pg} / \mathrm{ml}$, and the intraassay coefficients of variation were $5.5 \%$ for $183.9 \mathrm{pg} / \mathrm{ml}$ and $6.2 \%$ for $1537 \mathrm{pg} / \mathrm{ml}$. The interassay coefficients of variation were $7.5 \%$ for $181.02 \mathrm{pg} / \mathrm{ml}$ and $7.9 \%$ for $1547.9 \mathrm{pg} / \mathrm{ml}$.

Cell culture IFN- $\gamma$ concentrations were assayed using an ELISA kit from Biosource (Nivelles, Belgium) in duplicate. The mean of the two measurements was used as the final result. The analytical sensitivity of IFN- $\gamma$ was $173 \mathrm{pg} / \mathrm{ml}$, and the intraassay coefficients of variation were $1.6 \%$ for $290 \mathrm{pg} / \mathrm{ml}$ and $2.9 \%$ for $9.6 \mathrm{pg} / \mathrm{ml}$. The interassay coefficients of variation were $1.8 \%$ for 216 pg/ $\mathrm{ml}$ and $5.9 \%$ for $104 \mathrm{pg} / \mathrm{ml}$. 
Cell culture IL-17A concentrations were assayed using an ELISA kit from RayBio (Norcross, Georgia, USA) in duplicate. The mean of the two measurements was used as the final result. The analytical sensitivity of IL-17 was $10 \mathrm{pg} / \mathrm{ml}$, the intraassay coefficient of variation was $<10 \%$, and the interassay coefficient of variation was $<12 \%$.

Cell culture IL-23 concentrations were assayed using an ELISA kit from Bender MedSystems (Vienna, Austria, Europe) in duplicate. The mean of the two measurements was used as the final result. The analytical sensitivity of IL-23 was $10 \mathrm{pg} / \mathrm{ml}$, and the intraassay coefficients of variation were $3.8 \%$ for $414 \mathrm{pg} / \mathrm{ml}$ and $3.0 \%$ for $690 \mathrm{pg} /$ $\mathrm{ml}$. The interassay coefficients of variation were $3.7 \%$ for $401 \mathrm{pg} / \mathrm{ml}$ and $7.3 \%$ for $675 \mathrm{pg} / \mathrm{ml}$.

\section{Control group}

The control group comprised volunteers with similar ages and a similar gender distribution as the patient group who were students and staff of the Medical School and were not suffering from allergic complaints. These individuals underwent the same laboratory tests as the patients to exclude any inflammatory or autoimmune diseases. This group included 20 subjects with negative ASST results. A volume of $15 \mathrm{cc}$ of venous blood was collected from the control group subjects to perform PBMC.

\section{Statistical analysis}

The values are expressed as the mean \pm standard deviation (SD). The Statistical Package for Social Sciences (SPSS) Version 16.0 (Chicago, IL, USA) was used for statistical analysis. To compare the total patient group with the control group, the independent samples T test was used. To compare the ASST(+) and ASST(-) patient groups and the control group, the Kruskal-Wallis test was performed. The Mann-Whitney $U$ test was used to compare parameters between groups. For all tests, $p$-values of less than 0.5 were considered statistically significant.

\section{Results}

A total of 40 patients, including 29 (72.5\%) females and 11 (27.5\%) males who were diagnosed with CSU and were being treated at the Internal Diseases Allergy Immunology Clinic, participated in the study. The mean age of the patients was $38.2 \pm 10.4$ years (age range between 19 and 56). Twenty patients had positive ASST results, and 20 had negative results. The control group included 10 (50\%) males and 10 (50\%) females with a mean age of $36.5 \pm 5.45$ years (age range of between 18 and 58) and a total of 20 healthy subjects. The control group subjects had negative ASST results.

According to the UAS, the percentage of CSU patients with mild, moderate and severe urticaria were $17.5 \%$, $52.5 \%$ and $30.0 \%$, respectively. Among the ASST(+) patients, 3 (15\%) had mild disease, 8 (40\%) had moderate disease, and 9 (45\%) had severe disease, whereas among the ASST(-) patients, 4 (20\%) had mild disease, 13 (65\%) had moderate disease, and 3 (15\%) had severe disease. The ASST(+) patients had significantly higher UASs than the ASST $(-)$ patients $(p=0.021)$. Correlation analysis of the patient age, patient gender, UAS and cytokine level revealed no significant relationships.

Comparison of the total patient group with the control group without considering the ASST results revealed that the IL-23 ( $p=0.01), \mathrm{IL}-10(p=0.04)$ and IL-4 $(p=0.01)$ levels in the patient group were significantly lower.

The IL-4 level in the ASST(+) patient group was determined to be significantly higher than that of the ASST(-) patient group $(p=0.04)$.

The IL-23 $(p=0.009), \mathrm{IL}-10(p=0.009), \mathrm{IL}-4(p=0.001)$, and IL-17 ( $p=0.05)$ levels in the ASST(-) patient group were significantly lower compared with those in the control group.

The IL-4 ( $p=0.03)$ and IFN- $\gamma(p=0.05)$ levels in the ASST(+) patient group were significantly lower compared with those in the control group.

The comparison of cytokine levels in the ASST(+) patient group, the ASST(-) patient group, the total patient group and the control group and the corresponding p-values are shown in Figure 1.

\section{Discussion}

Only a small number of studies have examined the role of cytokines in the immunopathogenesis of CSU. In this study, the main objective was to contribute to the understanding of the pathogenesis of this condition by identifying its relationships with autoimmunity, T lymphocyte subgroups and cytokines by comparing patients suffering from CSU with healthy subjects. A secondary objective was to obtain results that could contribute to the development of new treatment approaches.

Initially, to differentiate patients with an autoimmune etiology, the ASST was used rather than the basophil secretion test, which is expensive and difficult to apply. The ASST has a higher sensitivity and specificity, is less expensive and is very safe compared with the basophil histamine secretion test. The ASST is the best in vivo test method, and its results are the most similar to those of the in vitro basophil histamine secretion test [7]. Additionally, PBMCs were performed to determine the cytokines that were only secreted by $T$ lymphocytes.

A total of 20 ASST(-) and 20 ASST(+) subjects and 40 CSU patients participated in this study, and PBMCS were performed to determine cytokine patterns. The IL-4, IL-10, TGF- $\beta$, IFN- $\gamma$, IL-17, and IL-23 levels in cell culture supernatants were measured by ELISA.

The IL-4 level of the patient group was significantly lower than that of the control group. In addition, the IL-4 levels of both the ASST(-) and ASST(+) patient groups were significantly lower compared with that of the control 

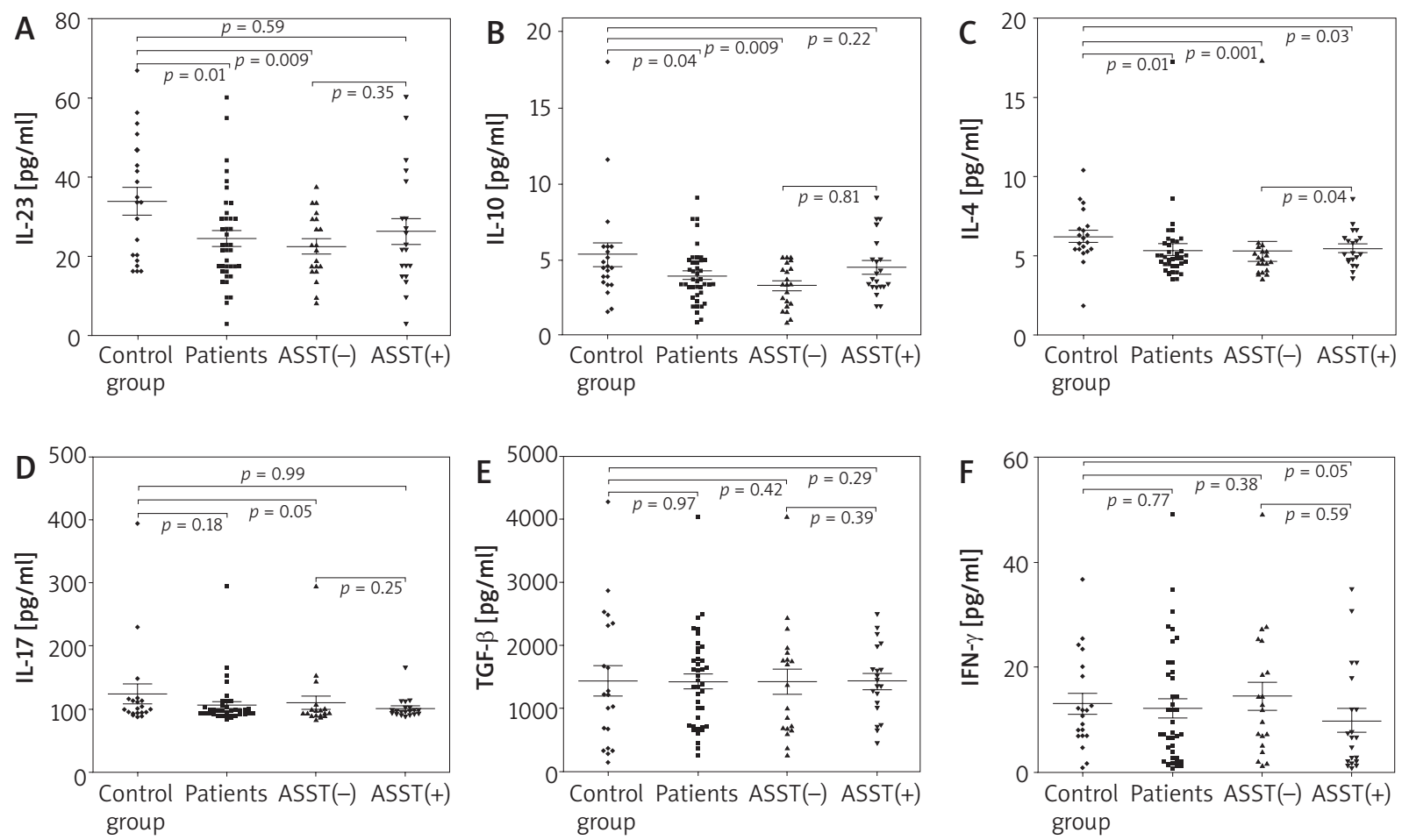

Figure 1 A-F. Comparison of cytokine levels of ASST(+) patient group, ASST(-) patient group, total patient group (patients), control group and $p$-values

group. Cohen et al. have found that IL-4 levels in PBMC supernatants of CSU patients are significantly lower compared with those of controls, similar to our findings [30]. Basophils are responsible for most of the stimulated IL-4 secretion in leukocyte cell cultures. It has been shown that basophils respond less to IgE-mediated and IgEunmediated stimulation in CSU patients [30, 31]. The low levels of IL-4 in CSU patients may be due to quantitative or functional defects in basophils. In both of the studies conducted by Cohen et al. and in our study, the basophil counts of the patient groups and control groups were normal, and basophils disappeared during PBMC. Similar to basophils, mast cells and lymphocytes secrete many cytokines, such as IL-4, that affect the cytokine balance in CSU patients. As a consequence, the reduced IL-4 secretion that was observed in the CSU patients in this study was associated with lymphocytes rather than basophils.

In accordance with three different studies in the literature, the IL-4 levels of the patients in our study were lower compared with those of the controls [29, 30, 32]. However, other previous studies have reported similar IL-4 levels between patients and controls [33, 34].

In the absence of IL-4, which is the major cytokine for Th2 differentiation, the Th1- mediated immune response is expected to be dominant. However, in our study and in the study conducted by Cohen et al., there was no increase in Th1-derived cytokines [30].

Two studies have shown that local Th2-mediated inflammation is dominant in skin biopsy samples from
CIU patients $[35,36]$. In our study, the Th2-derived cytokine IL-4, which is known to be abundant during local inflammation, was detected in systemic circulation. It is known that Th2-mediated inflammation and cytokines are predominant in all airways and in systemic circulation in diseases such as allergic rhinitis and asthma. By contrast, when thyroid cell cultures and peripheral blood cell cultures have been performed for autoimmune thyroid patients, significant differences have been observed between Th1 and Th2-derived cytokine levels $[37,38]$.

The current literature and our findings indicate that in CSU pathogenesis, although IgE-mediated reactions, Th2-mediated inflammation and Th2-derived cytokines play roles in local inflammation, they do not play considerable systemic roles.

In our study, the IL-10 levels of the patient groups were lower compared with those of the control group ( $p=0.04)$. In terms of the ASST, the IL-10 level of the ASST(-) patient group was significantly lower compared with that of the control group $(p=0.009)$. However, in two different studies, the IL-10 levels of CSU patients have been found to be higher compared with those of controls, in contrast with our findings [32-36]. In these studies, it has been suggested that IL-10 levels may have increased to control inflammation in these patients because it is an anti-inflammatory cytokine. However, Dos Santos et al. found that the IL-10 level of an ASST(+) patient group was higher compared with that of an ASST(-) patient 
group [38]. This finding suggests that autoantibodies in the sera of ASST(+) patients might affect PBMC results. However, further studies have found that the IL-10 levels of CSU patients are lower compared with those of controls, similar to our findings [30, 32]. Interleukin-10 is produced by dendritic cells, natural killer cells, B lymphocytes and Treg cells. It suppresses the antigen-specific immune response and reduces cytokine synthesis by T lymphocytes by deactivating macrophages. It can control Th2-mediated inflammation while inhibiting most of the functions of Th1 cells and macrophages. The IL-10 allows naive Th cells to differentiate into Th2 by suppressing IL-12 secretion from antigen presenting cells, but it suppresses the Th2 response in some cases resulting in proinflammatory cytokine and chemokine suppression. Because we used the PBMC method in our study, we know that the IL-10 level was directly associated with Treg cells. In conclusion, the fact that IL-10 production was decreased in CSU patients supports the hypothesis that there is insufficient Treg cell functioning.

In our study, the IFN- $\gamma$ level of the ASST(+) patient group was lower than that of the control group $(p=0.05)$. In addition, the mean IFN- $\gamma$ levels of the patient groups were lower than that of the control group, although this reduction was not statistically significant. Although three studies in the literature have found that the IFN- $\gamma$ levels of CSU patients are low, similar to our findings, no difference between patient and control groups has been observed in another study $[29,30,38]$. The low levels of IL-4 and IFN- $\gamma$ secretion observed in the CSU patients in our study might indicate that Th1 and Th2 functions are insufficient in these patients.

While there was not a statistically significant difference in the TGF- $\beta$ level between the patient and control groups in our study, the mean TGF- $\beta$ level of the patients was lower. In a study conducted by Chen et al., the TGF- $\beta$ levels of both patient and control groups were similar, in agreement with our findings [29]. Taken together, previous reports in the literature and our findings showing that IL-10 and TGF- $\beta$ levels are low in CSU patients support the notions that Treg cells are the source of IL-10 and that there is a defect in Treg cells in these patients.

In addition, a number of studies have indicated that Treg cells appear to control the development of autoimmune diseases and also play critical roles in controlling asthma and allergies [29, 30]. However, the characteristics and roles of these cells have not yet been defined in CSU patients. It is possible that these patients may have intrinsic abnormalities in Treg cells and that these deficient cells may be unable to inhibit the ongoing inflammation. Taken together, these findings indicate a generalized downregulation of both Th1 and Th2 cytokine production in CSU patients.

In our study, the IL-23 levels of the patient group were significantly lower than that of the control group $(p=0.01)$, particularly the ASST $(-)$ patient group ( $p=0.009)$.
The IL-17 was significantly lower in the ASST(-) patient group compared with the control group ( $p=0.05)$. In addition, the mean IL-17 level of the patient group was lower than that of the control group, although this finding was statistically insignificant. Dos Santos et al. have found that the IL-17 level of CU patients is higher than that of controls, in contrast with our findings [38].

In another study, Atwa et al. have found that serum concentrations of IL-17, IL-23 and tumor necrosis factor (TNF)- $\alpha$ are significantly higher in CSU patients compared with healthy control subjects [39].

Daschner et al. have found lower serum IL-17 levels in CSU patients with and without sensitization against Anisakis simplex A compared with those in patients without a history of urticaria [40]. The authors have suggested that the IL-17 level is diminished because of its consumption during the inflammatory process, similar to our findings.

Because the IL-23 and IL-17A levels were low in the patient groups in our study, it can be asserted that the Th17-dominant immune response is not very effective in CSU pathogenesis. A possible role of IL-17A in the pathology of inflammatory skin disorders, such as psoriasis, contact hypersensitivity and atopic dermatitis, has been described, indicating that it is an important mediator of tissue inflammation [41]. Thus, it might be relevant to verify whether Th17 cells are involved in CSU pathogenesis and the extent of their involvement.

In terms of the ASST, the fact that the IL-4, IL-10, IL-17A, and IL-23 levels in particular were significantly lower in the ASST(-) patient group compared with the control group suggests that Th2, Th17 and Treg cell functions were insufficient or suppressed in these patients.

To date, few studies have been carried out to define the cytokine profiles in PBMCs from CSU patients. In our study, the mean IL-4, IL-10, IFN- $\gamma$, TGF- $\beta$, IL-17A, and IL-23 levels of the patient groups were lower compared with those of the control group. These findings, in addition to previous reports in the literature, indicate that Th1, Th2, Th17 and Treg cell functions are suppressed or insufficient in CSU patients and that immune dysregulation occurs as part of the pathogenesis of CSU, resulting in cytokine imbalance.

The pathophysiology of CSU is complex, and it has not been well defined. Alterations in the cytokine profile of CSU patients and in Th1, Th2, Th17 and Treg cell cytokine levels in the serum and skin of these patients may be important in the pathophysiology of this condition.

However, there is a need for further studies of a larger number of patients.

\section{Conflict of interest}

The authors declare no conflict of interest. 


\section{References}

1. Grattan CE, Sabroe RA, Greaves MW. Chronic urticaria. J Am Acad Dermatol 2002; 146: 645-57.

2. Kaplan AP. Chronic urticaria: pathogenesis and treatment. J Allergy Clin Immunol 2004; 114: 465-74.

3. Greaves MW. Chronic idiopathic urticaria. Curr Opin Allergy Clin Immunol 2003; 3: 363-8.

4. Sabroe RA, Fiebiger E, Francis DM, et al. Classification of anti-FcepsilonRI and anti-lgE autoantibodies in chronic idiopatic urticaria and correlation with disease severity. J Allergy Clin Immunol 2000; 110: 492-9.

5. Sabroe RA, Grattan CE, Francis DM, et al. The autologous serum skin test: a screening test for antibodies in chronic idiopatic urticaria. Br J Dermatol 1999; 140: 446-52.

6. Sabroe RA, Greaves MW. Chronic idiopathic urticaria with functional autoantibodies: 12 years on. Br J Dermatol 2006; 154: 813-9.

7. Konstantinou G, Asero R, Maurer M, et al. EAACI/GA(2)LEN task force consensus report: the autologous serum skin test in urticaria. Allergy 2009; 64: 1256-68.

8. Taskapan O, Kutlu A, Karabudak O. Evaluation of autologous serum skin test results in patients with chronic idiopatic urticaria, allergic/nonallergic asthma or rhinitis and healthy people. Clin Exp Dermatol 2008; 33: 754-8.

9. Boguniewicz M. The autoimmune nature of chronic urticaria. Allergy Asthma Proc 2008; 29: 433-8.

10. Elias J, Boss E, Kaplan AP. Studies of the cellular infiltrate of chronic idiopathic urticaria: prominence of T-lymphocytes, monocytes, and mast cells. J Allergy Clin Immunol 1986; 78: 914-8.

11. Ying S, Kikuchi Y, Meng Q, et al. TH1/TH2 cytokines and inflammatory cells in skin biopsy specimens from patients with chronic idiopathic urticaria: comparison with the allergen-induced late-phase cutaneous reaction. J Allergy Clin Immunol 2002; 109: 694-700.

12. O'Shea JJ, Ma A, Lipsky P. Cytokines and autoimmunity. Nat Rev Immunol 2002; 2: 37-45.

13. Nelms K, Keegan AD, Zamorano J, et al. The IL-4 receptor: signaling mechanisms and biologic functions. Annu Rev Immunol 1999; 17: 701-38.

14. Paul WE, Zhu J. How are $T(H) 2$-type immune responses initiated and amplified? Nat Rev Immunol 2010; 10: 225-35.

15. Li MO, Flavell RA. TGF-beta: a master of all T cell trades. Cell 2008; 134: 392-404.

16. Konkel JE, Chen W. Balancing acts: the role of TGF-beta in the mucosal immune system. Trends Mol Med 2011; 17: 668-76.

17. Commins S, Steinke JW, Borish L. The extended IL-10 super family: IL-10, IL-19, IL-20, IL-22, IL-24, IL-26, IL-28, and IL-29. J Allergy Clin Immunol 2008; 121: 1108-11.

18. Murray PJ. The primary mechanism of the IL-10-regulated anti-inflammatory response is to selectively inhibit transcription. Proc Natl Acad Sci U S A 2005; 102: 8686-91.

19. Jutel M, Akdis M, Budak F, et al. IL-10 and TGF-beta cooperate in the regulatory $T$ cell response to mucosal allergens in normal immunity and specific immunotherapy. Eur J Immunol 2003; 33: 1205-14.

20. Akkoc T, de Koning PJ, Ruckert B, et al. Increased activationinduced cell death of high IFN-gamma-producing $\mathrm{T}(\mathrm{H}) 1$ cells as a mechanism of $\mathrm{T}(\mathrm{H}) 2$ predominance in atopic diseases. J Allergy Clin Immunol 2008; 121: 652-8.

21. Okamura H, Tsutsi H, Komatsu T, et al. Cloning of a new cytokine that induces IFN-gamma production by T cells. Nature 1995; 378: 88-91.

22. Arican O, Aral M, Sasmaz S, et al. Serum levels of TNF-alpha, IFN-gamma, IL-6, IL-8, IL-12, IL-17, and IL-18 in patients with active psoriasis and correlation with disease severity. Mediators Inflamm 2005; 2005: 273-9.
23. Bettelli E, Korn T, Oukka M, et al. Induction and effector functions of Th17 cells. Nature 2008; 453: 1051-7.

24. Miossec P, Korn T, Kuchroo VK. Interleukin-17 and type 17 helper T cells. N Engl J Med 2009; 361: 888-98.

25. Kastelein RA, Hunter CA, Cua DJ. Discovery and biology of IL-23 and IL-27: related but functionally distinct regulators of inflammation. Annu Rev Immunol 2007; 25: 221-42.

26. Beadling C, Slifka MK. Regulation of innate and adaptive immune responses by the related cytokines IL-12, IL-23, and IL-27. Arch Immunol Ther Exp (Warsz) 2006; 54: 15-24.

27. McGeachy MJ, Cua DJ. The link between IL-23 and Th17 cellmediated immune pathologies. Semin Immunol 2007; 19: 372-6.

28. Zuberbier T, Asero R, Bindslev-Jensen C, et al. Dermatology Section of the European Academy of Allergology and Clinical Immunology; Global Allergy and Asthma European Network; European Dermatology Forum; World Allergy Organization. EAACI/GA(2)LEN/EDF/WAO guideline: definition, classification and diagnosis of urticaria. Allergy 2009; 64: 1417-26.

29. Chen WC, Chiang BL, Liu HE, et al. Defective functions of circulating CD4+CD25+ and CD4+CD25-T cells in patients with chronic ordinary urticaria. J Dermatol Sci 2008; 51: 121-30.

30. Confino-Cohen R, Aharoni D, Goldberg A, et al. Evidence for aberrant regulation of the p21Ras pathway in PBMCs of patients with chronic idiopathic urticaria. J Allergy Clin Immunol 2002; 109: 349-56.

31. Claveau J, Lavoie A, Brunet C, et al. Comparison of histamine releasing factor recovered from skin and peripheral blood mononuclear cells of patients with chronic urticaria. Ann Allergy Asthma Immunol 1996; 77: 475-9.

32. Irinyi B, Aleksza M, Antal-Szalmás P, et al. Cytokine production of CD4+ and CD8+ peripheral T lymphocytes in patients with chronic idiopathic urticaria. Acta Derm Venereol 2002; 82: 249-53.

33. Piconi S, Trabattoni D, lemoli E, et al. Immune profiles of patients with chronic idiopathic urticaria. Int Arch Allergy Immunol 2002; 128: 59-66.

34. Caproni M, Cardinali C, Giomi B, et al. Serological detection of eotaxin, IL-4, IL-13, IFN-gamma, MIP-1alpha, TARC and IP-10 in chronic autoimmune urticaria and chronic idiopathic urticaria. J Dermatol Sci 2004; 36: 57-9.

35. Caproni M, Giomi B, Volpi W, et al. Chronic idiopathic urticaria: infiltrating cells and related cytokines in autologous serum-induced wheals. Clin Immunol 2005; 114: 284-92.

36. Ying S, Kikuchi Y, Meng Q, et al. Th1/Th2 cytokines and inflammatory cells in skin biopsy specimens from patients with chronic idiopathic urticaria: comparison with the allergen-induced late-phase cutaneous reaction. J Allergy Clin Immunol 2002; 109: 694-700.

37. Guo J, Rapoport B, McLachlan SM. Balance of Th1/Th2 cytokines in thyroid autoantibody synthesis in vitro. Autoimmunity 1999; 30: 1-9.

38. Dos Santos JC, Azor MH, Nojima VY, et al. Increased circulating pro-inflammatory cytokines and imbalanced regulatory T-cell cytokines production in chronic idiopathic urticaria. Int Immunopharmacol 2008; 8: 1433-40.

39. Atwa MA, Emara AS, Youssef N, et al. Serum concentration of IL-17, IL-23 and TNF-alpha among patients with chronic spontaneous urticaria: association with disease activity and autologous serum skin test. J Eur Acad Dermatol Venereol 2014; 28: 469-74.

40. Daschner A, Rodero M, De Frutos C, et al. Different serum cytokine levels in chronic vs. acute Anisakis simplex sensitization-associated urticaria. Parasite Immunol 2011; 33: 357-62.

41. van Beelen AJ, Teunissen MB, Kapsenberg ML, et al. Interleukin-17 in inflammatory skin disorders. Curr Opin Allergy Clin Immunol 2007; 7: 374-81. 